\title{
Identification of Citrus Canker on Citrus Leaves and Fruit Surfaces in the Grove Using Deep Learning Neural Networks
}

\author{
Matshwene Edwin Moshia ${ }^{1,2}$ and Loyiso Lloyd Mzini ${ }^{1}$ \\ 1. Department of Agronomy, University of Fort Hare, 1 King Williams Town Rd., Alice 5700, Eastern Cape, South Africa \\ 2. FAO-Intergovernmental Technical Panel on Soils, Vialle delle Terme de Caracalla, 00153, Rome, Italy
}

\begin{abstract}
Citrus canker (Xanthomonas axonopodis pv. citri) is a bacterial disease of citrus tree leaves and is the most feared citrus disease, affecting all types of important citrus crops. Currently, there is no cure for citrus canker but experimental prevention methods. Citrus canker is a quarantine disease, meaning that citrus fruits with citrus canker cannot be transported between countries or markets, and this is a huge threat to the citrus production industry. Citrus exports to international markets are highly restricted today due to fruit diseases such as citrus canker because fruits are quarantined for bigger world markets such as European Union (EU) and United States of America citrus markets. For a row-guided robot to detect citrus canker infections on leaves and fruits in the grove, there is a need for an accurate method that can detect defects on the leaves and surface of fruits, classify citrus leaf and fruit images on-the-go, and discriminate citrus canker from other defects. Previous methods in machine vision could detect defects on the surface of citrus fruits, though, the methods were unable to differentiate defects. The neural network system invents edge detection, and automatically learns to characterize the image in terms of edges that appear in the image, and give a more succinct, higher-level representation than raw pixels. A standard strategy in deep learning neural networks is to run the learning algorithm with many optimization parameters and pick the model that gives the best performance on a validation set. The authors are optimistic that identification of citrus canker on citrus leaves and fruit surfaces in the grove could be one of the agricultural problems that can potentially benefit from deep learning neural network approach, and consequently, help to eradicate the predicaments facing the citrus industry.
\end{abstract}

Key words: Artificial intelligence, automatic fruit detection, citrus canker, deep learning, precision agriculture.

\section{Introduction}

South Africa is among the world leading countries in commercial citrus production. According to Low Velder, a South African local newspaper, in terms of export volume and value, Southern Africa and Turkey compete for the position of the world's second largest citrus exporter with Spain leading in citrus export (https://lowvelder.co.za/445158/south-african-farmers -play-major-role-global-citrus-industry/). This year, 2019, the South African Department of Agriculture, Land Reform and Rural Development issued a media

Corresponding author: Matshwene Edwin Moshia, professor, research fields: precision agriculture, soil science and machine learning. statement about the arrival of the first shipment of citrus fruits in the breakbulk vessel into the People's Republic of China, which was celebrated as significant.

Citrus fruits are a major supplier of vitamin C. This vitamin $\mathrm{C}$ from citrus fruits has helped heal diseases such as scurvy, correct human blood pressure, and helped in wound healing to save a human life since the 1930s [1]. The study on child death because of early childhood scurvy or lack of enough ascorbic acid to meet metabolic requirements in infants was the most devastating study with a vital conclusion that vitamin $\mathrm{C}$ can save lives and help in wound healing [2]. Scurvy is a human disease caused by a deficiency of vitamin $\mathrm{C}$ in the body system. The scariest thing about 
human health and nutrition is that, apart from famine, this disease caused by vitamin $\mathrm{C}$ deficiency is undoubtedly the dietary deficiency disease that has instigated most suffering in recorded human history [3]. This reason alone, indisputably emphasizes the importance of the citrus industry to human survival, and therefore, the impact of the citrus industry on human health and saving of human lives cannot be overemphasized.

Fresh citrus fruits are known to be a good source of dietary fiber, which is linked with the prevention of gastrointestinal disease and lowering of circulating blood cholesterol [4]. The effect of citrus products on human health ranges from a significant reduction of obesity through weight loss in adults, which is one of the major causes of chronic diseases and consequently the death of more than 300,000 individuals per year $[5,6]$. Generally, lemons are rich in flavonoids, a significant part of a balanced nutriment, predominantly for their role in prevention of terminal human diseases, including heart diseases, and certain types of cancer [7-10]. Most importantly, in recent times, citrus fruit intake was associated with a reduction in chances of developing breast cancer [11]. Most importantly, citrus is a source of $\beta$-cryptoxanthin, which accumulates in mature lemon and is used in the treatment of colon cancer in combination with oxaliplatin [12-14]. While citrus fruits are valuable for their edible inner part of the fruit; in the lemon industry, a by-product called pectin is extracted and used in food industry and food processing as a gelling agent in pharmaceutical, and stabilizer in dairy products [15].

Although the citrus industry is directly linked to human health and survival, the unfortunate reality is that citrus industries of the major citrus producing regions of the world may soon or later shut down. This could happen because of terminal citrus diseases such as Huanglongbing (HLB) or citrus greening (Candidatus Liberibacter asiaticus), and citrus canker (Xanthomonas axonopodis pv. citri). These diseases are major threats to citrus industry worldwide including Brazil and Florida [16]. These diseases have a potential to cause devastating problems in citrus production and consequently economic losses in the agricultural industry worldwide [17]. Currently, there is no cure for citrus canker but experimental prevention methods. For this reason, monitoring and control of harmful infections and diseases of citrus trees such as citrus canker disease at an early stage are key to human health and survival.

\section{Challenges with Citrus Canker Disease}

Citrus canker is a bacterial disease of citrus tree leaves and is the most feared citrus disease, affecting all types of important citrus crops [18]. This bacterial disease, which affects citrus tree in the whole world, causes premature fruit drop on citrus trees resulting in trees producing no fruits [19]. In addition to trees producing no fruits, worse is that citrus canker disease can be spread rapidly by natural environmental factors such as the wind and rain, and anthropogenic factors such as landscaping equipment or people.

Citrus canker is a quarantine disease, meaning that citrus fruits with citrus canker cannot be transported between countries or markets, and this is a huge threat to the citrus production industry. Fruits that are infected by citrus canker are ugly, severely blemished, reduced quality and early maturity, and these could be one of the reasons that citrus canker infected fruits have no value in the market [18]. Citrus exports to international markets are highly restricted today due to fruit diseases such as citrus canker because fruits are quarantined for bigger world markets such as European Union (EU) and United States of America citrus markets [20]. What makes citrus canker a natural disaster of the citrus industry is that currently there is no effective method that can fully eliminate this disease. However, the basic strategy is to reduce the effect of infection and to prevent the spread [19].

Detecting citrus canker at the early stage of crop growth and development is a significant step to 
control this disease [21]. Accurate citrus fruit yield estimate before harvest is valuable to growers and decision makers [22]. Currently, the reliable method of identifying citrus canker in the field and packing house is through visual detection since most remote sensing methods are experimental, or either documented without validation [20]. Previous methods in remote sensing relied on taking aerial photographs, downloading or scanning the images into an image analysis software and then developing an algorithm to decide [23]. This is a time-demanding method that relies on a special skill of operating various software in image analysis that citrus producers lack.

\section{Deep Learning with Neural Networks}

For a row-guided robot to detect citrus canker infections on leaves and fruits in the grove, there is a need for an accurate method that can detect defects on the leaves and surface of fruits, classify citrus leaf and fruit images on-the-go, and discriminate citrus canker from other defects. Previous methods could detect defects on the surface of citrus fruits, though, the methods were unable to differentiate defects [24]. The aim is to achieve a near human level of identification of this disease on young and matured leaves as well as citrus fruits in the grove using innovative methods that are reliable.

An advanced and innovative form of machine learning, which qualifies computers to solve continuous problems such as citrus fruit disease by image recognition is progressively making an entry into the biological and agricultural sciences [25]. Recent developments in machine learning yielded new techniques to train deep neural networks [26], which has a potential to successfully recognize patterns of objects such as deficiencies on plant leaves either by color, structure and other morphological properties. In simple terms, a neural network is a computer model that seeks to emulate knowledge acquisition and organizational skills of how a human brain functions [27].
Deep neural networks have recently shown outstanding performance on image classification tasks [28]. The system invents edge detection, and automatically learns to characterize the image in terms of edges that appear in the image, and give a more succinct, higher-level representation than raw pixels. A standard strategy is to run the learning algorithm with many optimization parameters and pick the model that gives the best performance on a validation set [29]. These are called deep convolutional neural networks, which were established as a powerful set of models for image recognition problems [30]. Convolutional neural networks are a selection of neural structure that influences spatial relationships to reduce parameters that must be learned and thus improves upon general feed-forward backpropagation training [31]. Convolutional neural networks have received much attention in recent years because neural networks were proved capable of outperforming previous records in image recognition challenges [32].

Neural networks derive a model from training any kind of data, which qualifies neural networks to be suited for tasks with limited prior knowledge [33]. Rusk [25] reported that deep-learning methods use multiple processing layers to discover patterns and structure in very large data sets. In deep learning with a neural network, a single specific object can be differentiated from 1,000 different images since the system is trained from a large data [32]. Grinblat et al. [34] reported that there are agricultural and biological problems that are currently addressed through traditional machine vision techniques that could benefit from deep learning with a neural network approach. Most importantly, this method of deep learning neural network is less affected by natural variations such as changes in illumination, shadows, skewed leaves and occluded plants as compared to other previous image classification methods [32].

\section{Conclusions}

Deep neural network system requires a large set of 
image data. These large sets of data are trained for pattern complexity and to improve accuracy in classifying images of citrus canker on leaves and fruits. The deep neural network system takes much longer time to train data even on a faster Central Processing Unit (CPU), and therefore, deep learning neural networks need high-performance training Graphics Processing Unit (GPU) to finish work quickly. The key is to, (i) estimate the infections and fruit defects, (ii) estimate the number of fruits in the citrus grove and potential fruit size before harvesting, (iii) yield prediction, and (iii) make economic estimates such as planning of incomes, and calculation of net profit. There is a wide range of primary and secondary leaves defects that can resemble citrus canker infection but this method has a potential to differential such effects because deep neural network is not affected by natural variations such as changes in illumination, shadows, skewed leaves and occluded plants as compared to other previous image classification methods. Therefore, the authors are optimistic that identification of citrus canker on citrus leaves and fruit surfaces in the grove could be one of those agricultural problems that can potentially benefit from deep learning approach, and consequently, help to eradicate the predicaments facing the citrus industry.

\section{References}

[1] Crandon, J. H., Lund, C. C., and Dill, D. B. 1940. "Experimental Human Scurvy." New England Journal of Medicine 223: 353-69.

[2] Laxman, T. I., and Ingalls, T. I. 1937. "Vitamin C Deficiency and Wound Healing." Annals of Surgery 105 (4): 616-25

[3] Carpenter, K. J. 1988. The History of Scurvy and Vitamin $C$. New York: Cambridge University Press.

[4] Liu, Y., Heying, E., and Tanumihardjo, S. A. 2012. "History, Global Distribution, and Nutritional Importance of Citrus Fruits." Comprehensive Reviews in Food Science and Food Safety 11 (6): 530-45.

[5] Colker, C. M., Kaiman, D. S., Torina, G. C., Perlis, T., and Street, C. 1999. "Effects of Citrus aurantium Extract, Caffeine, and St. John's Wort on Body Fat Loss, Lipid Levels, and Mood States in Overweight Healthy Adults."
Current Therapeutic Research 60 (3): 145-53.

[6] Preuss, H. G., Di Ferdinando, D., Bagchi, M., and Bagchi, D. 2002. "Citrus Aurantium as a Thermogenic, Weight-Reduction Replacement for Ephedra: An Overview." Journal of Medicine 33 (1-4): 247-64.

[7] Del Rio, J. A., Fuster, M. D., Gómez, P., Porras, I., Garcia-Lidón, A., and Ortuño, A. 2004. "Citrus Limon: A Source of Flavonoids of Pharmaceutical Interest." Food Chemistry 84 (3): 457-61.

[8] Vanamala, J., Reddivari, L., Yoo, K. S., Pike, L. M., and Patil, B. S. 2006. "Variation in the Content of Bioactive Flavonoids in Different Brands of Orange and Grapefruit Juices." Journal of Food Composition and Analysis 19: 157-66.

[9] Lin, J., Rexrode, K. M., Hu, F., Albert, C. M., Chae, C. U., Rimm, E. B., Stampfer, M. J., and Manson, J. E. 2007. "Dietary Intakes of Flavonols and Flavones and Coronary Heart Disease in US Women." American Journal of Epidemiology 165 (11): 1305-13.

[10] Benavente-Garcia, O., and Castillo, J. 2008. "Update on Uses and Properties of Citrus Flavonoids: New Findings in Anticancer, Cardiovascular, and Anti-inflammatory Activity." Journal of Agricultural and Food Chemistry 56 (15): 6185-205.

[11] Song, J. K., and Bae, J. M. 2013. "Citrus Fruit Intake and Breast Cancer Risk: A Quantitative Systematic Review." Journal of Breast Cancer 16 (1): 72-6.

[12] González-Molina, E., Domínguez-Perles, R., Moreno, D. A., and García-Viguera, C. 2010. "Natural Bioactive Compounds of Citrus Limon for Food and Health." Journal of Pharmaceutical and Biomedical Analysis 51 (2): 327-45.

[13] San Millán, C., Soldevilla, B., Martín, P., Gil-Calderón, B., Compte, M., Pérez-Sacristán, B., Donoso, E., Peña, C., Romero, J., Granado-Lorencio, F., and Bonilla, F. 2015. " $\beta$-Cryptoxanthin Synergistically Enhances the Antitumoral Activity of Oxaliplatin through 8 NP73 Negative Regulation in Colon Cancer." Clinical Cancer Research 21 (19): 4398-409.

[14] Hernández-Alvarez, E., Blanco-Navarro, I., Pérez-Sacristán, B., Sánchez-Siles, L. M., and Granado Lorencio, F. 2016. "In Vitro Digestion-Assisted Development of a $\beta$-Cryptoxanthin-Rich Functional Beverage; in Vivo Validation Using Systemic Response and Faecal Content." Food Chemistry 208: 18-25.

[15] Schieber, A., Stintzing, F. C., and Carle, R. 2001. "By-Products of Plant Food Processing as a Source of Functional Compounds-Recent Developments." Trends in Food Science and Technology 12 (11): 401-13.

[16] Wetterich, C. B., Kumar, R., Sankaran, S., Junior, J. B., Eshani, R., and Marcassa, L. G. 2013. "A Comparative Study on Application of Computer Vision and 
Fluorescence Imaging Spectroscopy for Detection of Huanglongbing Citrus Disease in the USA and Brazil." Journal of Spectroscopy. Article 841738, 1-6.

[17] Dubey, S. R., and Jalal, A. S. 2014. "Automatic Fruit Disease Classification Using Images." Computer Vision and Image Processing in Intelligent Systems and Multimedia Technologies 1 (1): 82-100.

[18] Das, A. K. 2003. "Citrus Canker-A Review." Journal of Applied Horticulture 5 (1): 52-60.

[19] Zhang, M., and Meng, Q. 2011. "Automatic Citrus Canker Detection from Leaf Images Captured in Field." Pattern Recognition Letters 32 (15): 2036-46.

[20] Stegmayer, G., Milone, D. H., Garran, S., and Burdyn, L. 2013. "Automatic Recognition of Quarantine Citrus Diseases." Expert Systems with Applications 40 (9): 3512-7. Accessed August 30, 2019. http://fich.unl.edu.ar/sinc/sinc-publications/2013/SMGB1 3/sinc_SMGB12.pdf.

[21] Zhang, M., and Meng, Q. 2010. "Citrus Canker Detection Based on Leaf Images Analysis." In Proceedings of the 2nd International Conference on Information Science and Engineering, Hangzhou, China, 3584-7.

[22] Zhao, C., Lee, W. S., and He, D. 2016. "Immature Green Citrus Detection Based on Colour Feature and Sum of Absolute Transformed Difference (SATD) Using Colour Images in the Citrus Grove." Computers and Electronics in Agriculture 124: 243-53.

[23] Lillesand, T., Kiefer, R. W., and Chipman, J. 2014. Remote Sensing and Image Interpretation. Hoboken, NJ: John Wiley and Sons.

[24] Blasco, J., Aleixos, N., Gómez-Sanchis, J., and Moltó, E. 2009. "Recognition and Classification of External Skin Damage in Citrus Fruits Using Multispectral Data and Morphological Features." Biosystems Engineering 103 (2): 137-45.

[25] Rusk, N. 2016. "Deep Learning." Nature Methods 13 (1): 35.

[26] Kooi, T., Litjens, G., Van Ginneken, B., Gubern-Mérida,
A., Sánchez, C. I., Mann, R., den Heeten, A., and Karssemeijer, N. 2017. "Large Scale Deep Learning for Computer Aided Detection of Mammographic Lesions." Medical Image Analysis 35: 303-12.

[27] Goh, A. T. C. 1995. "Back-Propagation Neural Networks for Modeling Complex Systems." Artificial Intelligence in Engineering 9 (3): 143-51.

[28] Krizhevsky, A., Sutskever, I., and Hinton, G. E. 2012. "Image Net Classification with Deep Convolutional Neural Networks." Advances in Neural Information Processing Systems 25: 1097-105.

[29] Ngiam, J., Coates, A., Lahiri, A., Prochnow, B., Le, Q. V., and Ng, A. Y. 2011. "On Optimization Methods for Deep Learning." In Proceedings of the 28th International Conference on Machine Learning, 265-72.

[30] Karpathy, A., Toderici, G., Shetty, S., Leung, T., Sukthankar, R., and Li, F. F. 2014. "Large-Scale Video Classification with Convolutional Neural Networks." In Proceedings of the 2014 IEEE Conference on Computer Vision and Pattern Recognition, 1725-32.

[31] Arel, I., Rose, D. C., and Karnowski, T. P. 2010. "Deep Machine Learning-A New Frontier in Artificial Intelligence Research." Computational Intelligence Magazine 5 (4): 13-8.

[32] Dyrmann, M., Karstoft, H., and Midtiby, H. S. 2016. "Plant Species Classification Using Deep Convolutional Neural Network." Biosystems Engineering 151: 72-80.

[33] Behmann, J., Mahlein, A. K., Rumpf, T., Römer, C., and Plümer, L. 2015. "A Review of Advanced Machine Learning Methods for the Detection of Biotic Stress in Precision Crop Protection." Precision Agriculture 16 (3): 239-60.

[34] Grinblat, G. L., Uzal, L. C., Larese, M. G., and Granitto, P. M. 2016. "Deep Learning for Plant Identification Using Vein Morphological Patterns." Computers and Electronics in Agriculture 127: 418-24. 